\title{
An Improved FPT Algorithm for the Flip Distance Problem*
}

\author{
Shaohua Li $^{1}$, Qilong Feng ${ }^{2}$, Xiangzhong Meng ${ }^{3}$, and Jianxin Wang ${ }^{4}$ \\ 1 School of Information Science and Engineering, Central South University, \\ Changsha, Hunan, P. R. China \\ 2 School of Information Science and Engineering, Central South University, \\ Changsha, Hunan, P. R. China \\ 3 School of Information Science and Engineering, Central South University, \\ Changsha, Hunan, P. R. China \\ 4 School of Information Science and Engineering, Central South University, \\ Changsha, Hunan, P. R. China \\ jxwang@mail.csu.edu.cn
}

\begin{abstract}
Given a set $\mathcal{P}$ of points in the Euclidean plane and two triangulations of $\mathcal{P}$, the flip distance between these two triangulations is the minimum number of flips required to transform one triangulation into the other. The Parameterized Flip Distance problem is to decide if the flip distance between two given triangulations is equal to a given integer $k$. The previous best FPT algorithm runs in time $O^{*}\left(k \cdot c^{k}\right)\left(c \leq 2 \times 14^{11}\right)$, where each step has fourteen possible choices, and the length of the action sequence is bounded by $11 k$. By applying the backtracking strategy and analyzing the underlying property of the flip sequence, each step of our algorithm has only five possible choices. Based on an auxiliary graph $G$, we prove that the length of the action sequence for our algorithm is bounded by $2|G|$. As a result, we present an FPT algorithm running in time $O^{*}\left(k \cdot 32^{k}\right)$.
\end{abstract}

1998 ACM Subject Classification F.2.2 Geometrical Problems and Computations, G.2.1 Combinatorial Algorithms

Keywords and phrases triangulation, flip distance, FPT algorithm

Digital Object Identifier 10.4230/LIPIcs.MFCS.2017.65

\section{Introduction}

Given a set $\mathcal{P}$ of $n$ points in the Euclidean plane, a triangulation of $\mathcal{P}$ is a maximal planar subdivision whose vertex set is $\mathcal{P}[10]$. A flip operation to one diagonal $e$ of a convex quadrilateral in a triangulation is to remove $e$ and insert the other diagonal into this quadrilateral. Note that if the quadrilateral associated with $e$ is not convex, the flip operation is not allowed. The flip distance between two triangulations is the minimum number of flips required to transform one triangulation into the other.

Triangulations play an important role in computational geometry, which are applied in areas such as computer-aided geometric design and numerical analysis $[11,13,21]$.

Given a point set $\mathcal{P}$ in the Euclidean plane, we can construct a graph $G_{T}(\mathcal{P})$ in which every triangulation of $\mathcal{P}$ is represented by a vertex, and two vertices are adjacent if their

\footnotetext{
* This work is supported in part by the National Natural Science Foundation of China under Grants $(61420106009,61232001,61472449,61672536,61572414)$.
}

c) (i) () Shaohua Li, Qilong Feng, Xiangzhong Meng, and Jianxin Wang; licensed under Creative Commons License CC-BY

42nd International Symposium on Mathematical Foundations of Computer Science (MFCS 2017).

Editors: Kim G. Larsen, Hans L. Bodlaender, and Jean-Francois Raskin; Article No. 65; pp. 65:1-65:13

Leibniz International Proceedings in Informatics

LI I ICS Schloss Dagstuhl - Leibniz-Zentrum für Informatik, Dagstuhl Publishing, Germany 
corresponding triangulations can be transformed into each other through one flip operation. $G_{T}(\mathcal{P})$ is called the triangulations graph of $\mathcal{P}$. Properties of the triangulations graph are studied in the literature. Aichholzer et al. [1] showed that the lower bound of the number of vertices of $G_{T}(\mathcal{P})$ is $\Omega\left(2.33^{n}\right)$. Lawson and Charles [17] showed that the diameter of $G_{T}(\mathcal{P})$ is $O\left(n^{2}\right)$. Hurtado et al. [14] proved that the bound is tight. Since $G_{T}(\mathcal{P})$ is connected [17], any two triangulations of $\mathcal{P}$ can be transformed into each other through a certain number of flips.

The Flip Distance problem consists in computing the flip distance between two triangulations of $\mathcal{P}$, which was proved to be NP-complete by Lubiw and Pathak [18]. Pilz [20] showed that the Flip Distance problem is APX-hard. Aichholzer et al. [2] proved that the Flip Distance problem is NP-complete on triangulations of simple polygons. However, the complexity of the Flip Distance problem on triangulations of convex polygons has been open for many years, which is equivalent to the problem of computing the rotation distance between two rooted binary trees [23].

The Parameterized Flip Distance problem is: given two triangulations of a set of points in the plane and an integer $k$, deciding if the flip distance between these two triangulations is equal to $k$. For the Parameterized Flip Distance problem on triangulations of a convex polygon, Lucas [19] gave a kernel of size $2 k$ and an $O^{*}\left(k^{k}\right)$-time algorithm. Kanj and Xia [15] studied the Parameterized Flip Distance problem on triangulations of a set of points in the plane, and presented an $O^{*}\left(k \cdot c^{k}\right)$-time algorithm $\left(c \leq 2 \cdot 14^{11}\right)$, which applies to triangulations of general polygonal regions (even with holes or points inside it).

In this paper, we exploit the structure of the Parameterized Flip Distance problem further. At first, we give a nondeterministic construction process to illustrate our idea. The nondeterministic construction process contains only two types of actions, which are the moving action as well as the flipping and backing action. There are 4 choices for the moving action and one choice for the flipping and backing action. Given two triangulations and a parameter $k$, we prove that either there exists a sequence of actions of length at most $2 k$, following which we can transform one triangulation into the other, or we can reject this instance. Thus we get an improved $O^{*}\left(k \cdot 32^{k}\right)$-time FPT algorithm, which also applies to triangulations of general polygonal regions (even with holes or points inside it).

\section{Preliminaries}

In a triangulation $T$, a flip operation $f$ to an edge $e$ that is the diagonal of a convex quadrilateral $\mathcal{Q}$ is to delete $e$ and insert the other diagonal $e^{\prime}$ into $\mathcal{Q}$. We define $e$ as the underlying edge of $f$, denoted by $\varepsilon(f)$, and $e^{\prime}$ as the resulting edge of $f$, denoted by $\varphi(f)$. (For consistency and clarity, we continue to use some symbols and definitions from [15]). Note that if $e$ is not a diagonal of any convex quadrilateral in the triangulation, flipping $e$ is not allowed. Suppose that we perform a flip operation $f$ on a triangulation $T_{1}$ and get a new triangulation $T_{2}$. We say $f$ transforms $T_{1}$ into $T_{2} . T_{1}$ is called an underlying triangulation of $f$, and $T_{2}$ is called a resulting triangulation of $f$. Given a set $\mathcal{P}$ of $n$ points in the Euclidean plane, let $T_{\text {start }}$ and $T_{\text {end }}$ be two triangulations of $\mathcal{P}$, in which $T_{\text {start }}$ is the initial triangulation and $T_{\text {end }}$ is the objective triangulation. Let $F=\left\langle f_{1}, f_{2}, \ldots, f_{r}\right\rangle$ be a sequence of flips, and $\left\langle T_{0}, T_{1}, \ldots, T_{r}\right\rangle$ be a sequence of triangulations of $\mathcal{P}$ in which $T_{0}=T_{\text {start }}$ and $T_{r}=T_{\text {end }}$. If $T_{i-1}$ is an underlying triangulation of $f_{i}$, and $T_{i}$ is a resulting triangulation of $f_{i}$ for each $i=1,2, \ldots, r$, we say $F$ transforms $T_{\text {start }}$ into $T_{\text {end }}$, or $F$ is a valid sequence, denoted by $T_{\text {start }} \stackrel{F}{\rightarrow} T_{\text {end }}$. The flip distance between $T_{\text {start }}$ and $T_{\text {end }}$ is the length of a shortest valid flip sequence. 
Now we give the formal definition of the Parameterized Flip Distance problem.

Parameterized Flip Distance

Input: Two triangulations $T_{\text {start }}$ and $T_{\text {end }}$ of $\mathcal{P}$ and an integer $\mathrm{k}$.

Question: Decide if the flip distance between $T_{\text {start }}$ and $T_{\text {end }}$ is equal to $k$.

The triangulation on which we are performing a flip operation is called the current triangulation. An edge $e$ which belongs to the current triangulation but does not belong to $T_{\text {end }}$ is called a necessary edge in the current triangulation. It is easy to see that for any necessary edge $e$, there must exist a flip operation $f$ in a valid sequence such that $e=\varepsilon(f)$. Otherwise, we cannot get the objective triangulation $T_{\text {end }}$.

For a directed graph $D$, a maximal connected component of its underlying graph is called a weakly connected component of $D$. We define the size of an undirected tree as the number of its vertices.

A parameterized problem is a decision problem for which every instance is of the form $(x, k)$, where $x$ is the input instance and $k \in \mathbb{N}$ is the parameter. A parameterized problem is fixed-parameter tractable (FPT) if it can be solved by an algorithm (FPT algorithm) in $O\left(f(k)|x|^{O(1)}\right)$ time, where $f(k)$ is a computable function of $k$. In addition to computational geometry, parameterized problems in other areas such as graph theory $[8,9,12]$, computational biology [3, 22] and MAX-SAT [6] are also studied extensively. For a further introduction to parameterized algorithms, readers could refer to $[4,7]$.

\section{The Improved Algorithm for the Parameterized Flip Distance Problem}

Given $T_{\text {start }}$ and $T_{\text {end }}$, let $F=\left\langle f_{1}, f_{2}, \ldots, f_{r}\right\rangle$ be a valid sequence, that is, $T_{\text {start }} \stackrel{F}{\rightarrow} T_{\text {end }}$. Definition 1 defines the adjacency of two flips in $F$.

- Definition 1. [15] Let $f_{i}$ and $f_{j}$ be two flips in $F(1 \leq i<j \leq r)$. We define that flip $f_{j}$ is adjacent to flip $f_{i}$, denoted by $f_{i} \rightarrow f_{j}$, if the following two conditions are satisfied:

(1) either $\varphi\left(f_{i}\right)=\varepsilon\left(f_{j}\right)$, or $\varphi\left(f_{i}\right)$ and $\varepsilon\left(f_{j}\right)$ share a triangle in triangulation $T_{j-1}$;

(2) $\varphi\left(f_{i}\right)$ is not flipped between $f_{i}$ and $f_{j}$, that is, there does not exist a flip $f_{p}$ in $F$, where $i<p<j$, such that $\varphi\left(f_{i}\right)=\varepsilon\left(f_{p}\right)$.

By Definition 1, we can construct a directed acyclic graph (DAG), denoted by $D_{F}$. Every node in $D_{F}$ represents a flip operation of $F$, and there is an $\operatorname{arc}$ from $f_{i}$ to $f_{j}$ if $f_{j}$ is adjacent to $f_{i}$. For convenience, we label the nodes in $D_{F}$ using labels of the corresponding flip operations. In other words, we can see a node in $D_{F}$ as a flip operation and vice versa.

The following lemma shows that any topological sorting of $D_{F}$ is a valid sequence.

- Lemma 2. [15] Let $T_{0}$ and $T_{r}$ be two triangulations and $F=\left\langle f_{1}, f_{2}, \ldots, f_{r}\right\rangle$ be a sequence of flips such that $T_{0} \stackrel{F}{\rightarrow} T_{r}$. Let $\pi(F)$ be a permutation of the flips in $F$ such that $\pi(F)$ is a topological sorting of $D_{F}$. Then $\pi(F)$ is a valid sequence of flips such that $T_{0} \stackrel{\pi(F)}{\longrightarrow} T_{r}$.

Lemma 2 ensures that if we repeatedly remove a source node from $D_{F}$ and flip the underlying edge of this node until $D_{F}$ becomes empty, we can get a valid sequence and the objective triangulation $T_{\text {end }}$.

Here we introduce the definition of a walk.

- Definition 3. [16] A walk in a triangulation $T$ (starting from an edge $e \in T$ ) is a sequence of edges of $T$ beginning with $e$ in which any two consecutive edges share a triangle in $T$. 
According to Lemma 2 , if there is a valid sequence $F$ for the input instance, any topological sorting of $D_{F}$ is also a valid sequence for the given instance. The difficulty is that $F$ is unknown. In order to find the topological sorting of $D_{F}$, the algorithm in [15] takes a nondeterministic walk to find an edge $e$ which is the underlying edge of a source node, flips this edge (removing the corresponding node from $D_{F}$ ), nondeterministically walks to an edge which shares a triangle with $e$ and recursively searches for an edge corresponding to a source node. They deal with weakly connected components of $D_{F}$ one after one (refer to Corollary 4 in [15]), that is, their algorithm tries to find a solution $F$ in which all flips belonging to the same weakly connected component of $D_{F}$ appear consecutively. In order to keep searching procedure within the current weakly connected component, they use a stack to preserve the nodes (defined as connecting point in [15]) whose removal separates the current weakly connected component into small weakly connected components. When removing all nodes of a small component, their algorithm jumps to the connecting point at the top of the stack and moves to deal with another small component.

We observe that it is not necessary to remove all nodes of a weakly connected component before dealing with other weakly connected components, that is, our algorithm may find a solution $F$ in which the nodes belonging to the same weakly connected components appear dispersedly. Thus we do not need a stack to preserve connecting points. Instead of five types of actions in [15], we only need two types, that is, moving action as well as flipping and backtracking action (see Section 3.2). Moreover, every time we find a source node, we remove the node, flip the underlying edge and backtrack instead of searching for the next node in four directions, thus reducing the number of choices for the actions. Another contribution of this paper is that we construct an auxiliary graph $G$ and prove that $G$ is a forest. Since there is a bijection between nondeterministic actions and nodes as well as edges of $G$, we prove that there exists a sequence of actions of length at most $2\left|D_{F}\right|$, which is smaller than $11\left|D_{F}\right|$ in [15]. In addition, we make some optimization on the strategy of finding the objective sequence. As a result, we improve the running time of the algorithm from $O^{*}\left(k \cdot c^{k}\right)$ where $c \leq 2 \cdot 14^{11}$ to $O^{*}\left(k \cdot 32^{k}\right)$.

\subsection{Nondeterministic construction process}

Now we give a description of our nondeterministic construction process NDTRV (see Fig. 1). The construction is nondeterministic as it always guesses the optimal choice correctly when running. The actual deterministic algorithm enumerates all possible choices to simulate the nondeterministic actions (see Fig. 3). Readers could refer to [5] as an example of nondeterministic algorithm. We present this construction process in order to depict the idea behind our deterministic algorithm clearly and vividly.

Let $T_{\text {start }}$ be the initial triangulation, and $T_{\text {end }}$ be the objective triangulation. Suppose that $F$ is a shortest valid sequence, that is, $F$ has the shortest length among all valid sequences. Let $D_{F}$ be the DAG constructed after $F$ according to Definition 1. NDTRV traverses $D_{F}$, removes the vertices of $D_{F}$ in a topologically-sorted order and transforms $T_{\text {start }}$ into $T_{\text {end }}$. Although $D_{F}$ is unknown, for further analysis, we assume that NDTRV can remove and copy nodes in $D_{F}$ so that it can construct an auxiliary undirected graph $G$ and a list $L$ based on $D_{F}$ during the traversal. In later analysis we show that $G$ is a forest, and there is a bijection between actions of NDTRV and nodes as well as edges of $G$. Obviously $G$ and $L$ are unknown as well. We just show that if a shortest valid sequence $F$ exists, then $D_{F}$ exists. So do $G, L$ and $Q$. We can see $D_{F}$ and $G$ as conceptual or dummy graphs. We construct $G$ instead of analysing a subgraph of $D_{F}$ because one move action (see Section 3.2) of NDTRV may correspond to one or two edges in $D_{F}$ (see Fig. 2), while there is a one-to-one correspondence between move actions and edges in $G$. 
At the beginning of an iteration, NDTRV picks a necessary edge $e=\varepsilon\left(f_{h}\right)$ arbitrarily and nondeterministically guesses a walk $W$ to find the underlying edge of a source node $f_{s}$. Lemma 5 shows that there exists such a walk $W$ whose length is bounded by the length of a directed path $B$ from $f_{s}$ to $f_{h}$, and every edge $e^{\prime}$ in $W$ is the underlying edge of some flip $f^{\prime}$ on $B$. NDTRV uses $L$ to preserve a sequence of nodes $\Gamma=\left\langle f_{s}=v_{1}, \ldots, f_{h}=v_{\ell}\right\rangle$ on $B$, whose underlying edges are in $W$. Simultaneously NDTRV constructs a path $S$ by copying all nodes in $\Gamma$ as well as adding an undirected edge between the copy of $v_{i}$ and $v_{i+1}$ for $i=1, \ldots, \ell . S$ is defined as a searching path. The node $f_{h}$ is called a starting node. If a starting node is precisely a source node in $D_{F}$, the searching path consists only of the copy of this starting node. When finding $\varepsilon\left(f_{s}\right)$, NDTRV removes $f_{s}$ from $D_{F}$, flips $\varepsilon\left(f_{s}\right)$ and moves back to the previous edge $\varepsilon\left(v_{2}\right)$ of $\varepsilon\left(f_{s}\right)$ in $W$. If $v_{2}$ becomes a source node of $D_{F}$, NDTRV removes $v_{2}$ from $D_{F}$, flips $\varepsilon\left(v_{2}\right)$ and moves back to the previous edge $\varepsilon\left(v_{3}\right)$. NDTRV repeats the above operations until finding a node $v_{i}$ in $\Gamma$ which is not a source node in $D_{F}$. Then NDTRV uses $v_{i}$ as a new starting node, and recursively guesses a walk nondeterministically from $\varepsilon\left(v_{i}\right)$ to find another edge which is the underlying edge of a source node as above. NDTRV performs these operations until the initial starting node $f_{h}$ becomes a source node in $D_{F}$. Finally NDTRV removes $f_{h}$ and flips $\varepsilon\left(f_{h}\right)$, terminating this iteration. NDTRV repeats the above iteration until $T_{\text {start }}$ is transformed into $T_{\text {end }}$. We give the formal presentation of NDTRV in Fig. 1 and an example in Fig. 2.

\subsection{Actions of the construction}

Our construction process contains two types of actions operating on triangulations. The edge which the algorithm is operating on is called the current edge. The current triangulation is denoted by $T_{\text {current }}$.

(i) Move to one edge that shares a triangle with the current edge in $T_{\text {current }}$. We formalize it as (move, $e_{1} \mapsto e_{2}$ ), where $e_{1}$ is the current edge and $e_{2}$ shares a triangle with $e_{1}$.

(ii) Flip the current edge and move back to the previous edge of the current edge in $W$. We formalize it as $\left(f, e_{4} \mapsto e_{3}\right)$, where $f$ is the flip performed on the current edge, $e_{4}$ equals $\varphi(f)$ and $e_{3}$ is the previous edge of $\varepsilon(f)$ in the current walk $W$.

Since there are four edges that share a triangle with the current edge, there are at most four directions for an action of type (i). However, there is only one choice for an action of type (ii).

\subsection{The sequence of actions}

The following theorem is the main theorem for the deterministic algorithm FLIPDT, which bounds the length of the sequence of actions by $2\left|V\left(D_{F}\right)\right|$.

- Theorem 4. There exists a sequence of actions of length at most $2\left|V\left(D_{F}\right)\right|$ following which we can perform a sequence of flips $F^{\prime}$ of length $\left|V\left(D_{F}\right)\right|$, starting from a necessary edge in $T_{\text {start }}$, such that $F^{\prime}$ is a topological sorting of $D_{F}$.

In order to prove theorem 4 , we need to introduce some lemmas. We will give the proof for Theorem 4 at the end of Section 3.3.

Lemma 5 shows the existence of a length-bounded walk to find an edge which is the underlying edge of a source node.

- Lemma 5. [16] Suppose that a sequence of flips $F^{-}$is performed such that every time we flip an edge, we delete the corresponding source node in the DAG resulting from preceding 
$\operatorname{NDTRV}\left(T_{\text {start }}, T_{\text {end }} ; D_{F}\right)$

Input: the initial triangulation $T_{\text {start }}$ and objective triangulation $T_{\text {end }}$. Assuming $F$ is a shortest sequence, $D_{F}$ is the corresponding DAG according to Definition 1.

$/{ }^{*} G$ is an auxiliary undirected graph */

$/{ }^{*} L$ is a list keeping track of searching paths for backtracking */

$/ * Q$ is a list preserving the sequence of nondeterministic actions */

/* $T_{\text {current }}$ is the current triangulation*/

a. Let $V(G)$ and $E(G)$ be empty sets, $L$ and $Q$ be empty lists;

b. $T_{\text {current }}=T_{\text {start }}$;

c. While $T_{\text {current }} \neq T_{\text {end }}$ do

c.1. Pick a necessary edge $e=\varepsilon\left(f_{h}\right)$ in $T_{\text {current }}$ arbitrarily;

c.2. Add a copy of $f_{h}$ into $G$;

c.3. Add $f_{h}$ into $L$;

c.4. TrackTree $\left(T_{\text {current }}, e, D_{F}, G, L, Q\right)$;

TrackTree $\left(T_{\text {current }}, \varepsilon\left(f_{h}\right), D_{F}, G, L, Q\right) /{ }^{*}$ construct searching paths starting from $\varepsilon\left(f_{h}\right) * /$

1. Nondeterministically guess a walk in $T_{\text {current }}$ from $\varepsilon\left(f_{h}\right)$ to find $\varepsilon\left(f_{s}\right)$ according to

Lemma 5 , let $\Gamma=\left\langle f_{s}=v_{1}, \ldots, f_{h}=v_{\ell}\right\rangle$, where $f_{s}$ is a source node in $D_{F}$, be a sequence of nodes on the backbone $B$ whose underlying edges are in the walk $W$ such that $\varepsilon\left(v_{i}\right)$ and $\varepsilon\left(v_{i+1}\right)$ are consecutive in $W$ for $i=1, \ldots, \ell-1$;

2. Add a copy of $v_{1}, \ldots, v_{\ell-1}$ into $G$ respectively;

3. Connect the copies of $v_{1}, \ldots, v_{\ell}$ in $G$ into a path;

4. Append $v_{\ell-1}, \ldots, v_{1}$ to $L$; $\quad /{ }^{*}$ record current searching path*/

5. Append (move, $\left.\varepsilon\left(v_{\ell}\right) \mapsto \varepsilon\left(v_{\ell-1}\right)\right), \ldots,\left(\right.$ move, $\left.\varepsilon\left(v_{2}\right) \mapsto \varepsilon\left(v_{1}\right)\right)$ to $Q ; \quad /{ }^{*}$ record actions* ${ }^{*} /$

6. Remove $f_{s}=v_{1}$ from $L$

7. Remove $f_{s}=v_{1}$ from $D_{F}$;

8. Flip $\varepsilon\left(f_{s}\right)$ in $T_{\text {current }}$ and move back to $\varepsilon\left(v_{2}\right)$;

9. Append $\left(f_{s}, \varphi\left(v_{1}\right) \mapsto \varepsilon\left(v_{2}\right)\right)$ to $Q ; \quad /{ }^{*}$ record actions*/

10. For $i=2$ to $\ell$ do

10.1 Nondeterministically guess if $v_{i}$ is a source node in $D_{F}$;

10.2 If $v_{i}$ is a source node of $D_{F}$ then /*flip and move back*/

10.2.1 Remove $v_{i}$ from $L$;

10.2.2 Remove $v_{i}$ from $D_{F}$;

10.2.3 Flip $\varepsilon\left(v_{i}\right)$ in $T_{\text {current }}$ and move back to $\varepsilon\left(v_{i+1}\right)$;

10.2.4 Append $\left(v_{i}, \varphi\left(v_{i}\right) \mapsto \varepsilon\left(v_{i+1}\right)\right)$ to $Q$;

10.3 Else $\quad /{ }^{*}$ construct searching paths from $v_{i}{ }^{*} /$

10.3.1 TrackTree $\left(T_{\text {current }}, \varepsilon\left(v_{i}\right), D_{F}, G, L, Q\right)$;

Figure 1 Nondeterministic construction NDTRV

deleting operations. Let $f_{h}$ be a node in the remaining DAG such that $\varepsilon\left(f_{h}\right)$ is an edge in the triangulation $T$ resulting from performing the sequence of flips $F^{-}$. There is a source node $f_{s}$ in the remaining $D A G$ satisfying:

(1) There is a walk $W$ in $T$ from $\varepsilon\left(f_{h}\right)$ to $\varepsilon\left(f_{s}\right)$.

(2) There is a directed path $B$ from $f_{s}$ to $f_{h}$ in the remaining DAG that we refer to as the backbone of the DAG.

(3) The length of $W$ is at most that of $B$.

(4) Any edge in $W$ is the underlying edge of a flip in $B$, that is, $W=\left\langle\varepsilon\left(v_{1}\right), \ldots, \varepsilon\left(v_{\ell}\right)\right\rangle$, where $v_{1}=f_{s}, \ldots, v_{\ell}=f_{h}$ are nodes in $B$ and there is a directed path $B_{i}$ from $v_{i}$ to $v_{i+1}$ for $i=1, \ldots, \ell-1$ such that $B_{i} \subset B$. A searching path is an undirected path constructed by copying $v_{1}, \ldots, v_{\ell}$ and connecting the copies of $v_{1}, \ldots, v_{\ell}$ into a path.

- Lemma 6. NDTRV transforms $T_{\text {start }}$ into $T_{\text {end }}$ with the minimum number of flips and stops in polynomial time if it correctly guesses every moving and flipping action.

Proof. Suppose that $F$ is a shortest valid sequence. According to Lemma 5, every edge 

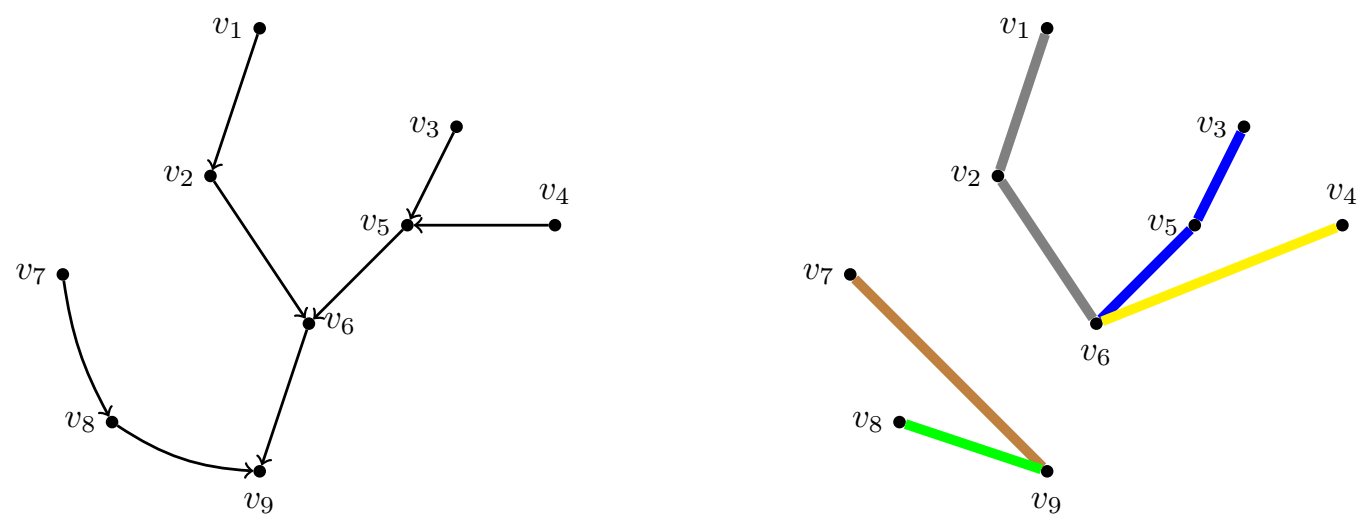

Figure 2 An example for constructing $G$. The graph on the left is the DAG. The graph on the right is the auxiliary graph $G$ constructed by NDTRV. $\varepsilon\left(v_{6}\right)$ is the first chosen necessary edge, and $\varepsilon\left(v_{9}\right)$ is the second one. Edges with the same color belong to the same searching path. Searching paths in the same connected component are constructed in the same iteration.

flipped in NDTRV is the underlying edge of a source node in the remaining graph of $D_{F}$, and every node removed from the remaining graph of $D_{F}$ in NDTRV is a source node. If $T_{\text {current }}$ is equal to $T_{\text {end }}$ but $D_{F}$ is not empty, then there exists a valid sequence $F^{\prime}$ which is shorter than $F$, contradicting that $F$ is a shortest valid sequence. Thus NDTRV traverses $D_{F}$, removes all nodes of $D_{F}$ in a topologically-sorted order and transforms $T_{\text {start }}$ into $T_{\text {end }}$ with the minimum number of flips by Lemma 2. Since the diameter of a transformations graph $G_{T}(\mathcal{P})$ is $O\left(n^{2}\right)$ [17], NDTRV stops in polynomial time.

NDTRV constructs $G, Q$ and $L$ during its execution. Lemma 7, Lemma 8 and Lemma 9 show some properties of $G, Q, L$ and $D_{F}$.

- Lemma 7. At the end of NDTRV, the graph $G$ consists of all searching paths constructed during the execution of $\mathbf{N D T R V}$. Moreover, the list $Q$ contains a sequence of nondeterministic actions starting from a necessary edge in $T_{\text {start }}$ following which we can perform a sequence of flips $F^{\prime}$ of length $\left|V\left(D_{F}\right)\right|$ such that $F^{\prime}$ is a topological sorting of $D_{F}$.

Proof. From the construction of $G$, we see that one node is added into $G$ when it is in some searching path. Moreover, we add an edge between two nodes in $G$ if and only if they are adjacent in a searching path. It follows that $G$ consists of all searching paths constructed during the execution of NDTRV.

By the proof of Lemma 6 , NDTRV removes the nodes of $D_{F}$ in a topologically-sorted order. Since $Q$ records every action of NDTRV, if we perform the actions in $Q$, we get a valid sequence $F^{\prime}$. This completes the proof.

- Lemma 8. The following statements are true:

(1) At the end of any iteration of NDTRV, all nodes whose copies are in the searching paths constructed in this iteration are removed from $D_{F}$, and $L$ becomes empty.

(2) During every step of NDTRV, there exists a directed path in the remaining graph of $D_{F}$ passing through every node in $L$ from the last node of $L$ to the first node of $L$.

(3) All searching paths in $G$ are edge-disjoint. Any two searching paths which belong to two different iterations respectively are node-disjoint. Searching paths constructed in each iteration form a tree respectively, namely a track tree. Moreover, $G$ is a forest. 
Proof. Suppose we are in the first iteration. We name the searching paths in this iteration $S_{1}, \ldots, S_{m}$ by the order they are constructed. Suppose now we have constructed searching paths $S_{1}, \ldots, S_{i}$ and $i<m$. We prove that statements (2) and (3) hold for the first iteration by induction on $i$. When $i=1$, the statement is true because there is only one searching path $S_{1}$ forming a tree, and there is a directed path in the remaining graph of $D_{F}$ passing through every node in $L$ from the last to the first according to Lemma 5 . Assume that the statements are true for any $i<m$, that is, $S_{1}, \ldots, S_{i}$ are edge-disjoint, and they form a tree. Moreover, there exists a directed path $P$ in the remaining graph of $D_{F}$ passing through every node in $L$ from the last to the first (inductive hypothesis). The next searching path we will construct is $S_{i+1}$. Note that before constructing $S_{i+1}$, we may remove the last node of $L$ repeatedly from the remaining graph of $D_{F}$ and $L$ if it is a source node in the remaining graph of $D_{F}$. Since every node we removed was the beginning node of the directed path $P$, there was still a directed path passing through every node in $L$ from the last to the first. Let $f_{\text {start }, i+1}$ be the starting node whose copy is in $S_{i+1}$, which means that $f_{\text {start }, i+1}$ is the last node in $L$, and $f_{\text {start }, i+1}$ is not a source node in the remaining graph of $D_{F}$. By the inductive hypothesis and the analysis above, there is a directed path $P_{1}$ from $f_{\text {start }, i+1}$ to $f_{h}$ which is the first node of $L$. Let $f_{s}^{\prime}$ be a source node in the remaining graph of $D_{F}$ such that the copy of $f_{s}^{\prime}$ is in $S_{i+1}$. By Lemma 5 , there is a directed path $P_{2}$ in the remaining graph of $D_{F}$ passing through the nodes whose copies are in $S_{i+1}$ from $f_{s}^{\prime}$ to $f_{\text {start }, i+1}$. We argue that $P_{1}$ has only one common node with $P_{2}$, which is exactly $f_{\text {start }, i+1}$. Otherwise, there is a directed cycle induced by some of the nodes on $P_{1}$ and $P_{2}$ in the remaining graph of $D_{F}$, contradicting the acyclicity of $D_{F}$. It follows that there is a directed path in the remaining graph of $D_{F}$ passing through every node in $L$ from the last node of $L$ to the first node of $L$. Since $S_{i+1}$ cannot contain any copies of the nodes that have been removed from the remaining graph of $D_{F}$ and $L, S_{i+1}$ has only one common node with the searching paths $S_{1}, \ldots, S_{i}$, namely the copy of $f_{\text {start }, i+1}$. It is implied that a node of $D_{F}$ cannot be added to $L$ more than once for the same reason. As a result, $S_{1}, \ldots, S_{i}$ and $S_{i+1}$ are edge-disjoint, and $S_{1}, \ldots, S_{i+1}$ form a tree. The tree formed by $S_{1}, \ldots, S_{m}$ is a track tree. This completes the inductive proof.

We prove statement (1) in the first iteration by contradiction. Suppose that there exists one or several nodes left in $L$ at the end of an iteration, and $w$ is the last one in $L$. Suppose $w$ was added to $L$ when searching path $S_{p}$ was constructed and $\operatorname{TrackTree}\left(v_{p}\right)$ was called. Let $u$ be the node appended to $L$ on its heel. Such a node $u$ existed since $w$ was not a source node then. Otherwise $w$ was removed, resulting in a contradiction as one node of $D_{F}$ cannot be added to $L$ more than once (according to the proof in the above paragraph). Since $w$ is the last one in $L$ at the end of this iteration, $u$ was removed, and NDTRV moved back to $\varepsilon(w)$. According to NDTRV, $w$ has never become a source node in $D_{F}$. Otherwise, $w$ has been removed from $L$, leading to a contradiction since one node of $D_{F}$ cannot be added to $L$ more than once. Thus $\operatorname{Track} \operatorname{Tree}(w)$ was called. The terminal condition of $\operatorname{TrackTree}(w)$ is that $w$ becomes a source node in $D_{F}$, and $w$ is removed from $L$ and $D_{F}$. This implies that this iteration does not end, a contradiction. It follows that $L$ becomes empty at the end of the first iteration. According to NDTRV, all nodes whose copies are in the searching paths constructed in the first iteration are removed from $D_{F}$.

We prove that statements (1), (2) and (3) hold for the whole procedure by induction. We have proved that they are true for the first iteration. Suppose that there are $t$ iterations in the procedure, and statements (1), (2) and (3) hold for the first $j$ iterations for any $1 \leq j<t$. By the inductive hypothesis, at the end of the $j$-th iteration, $L$ is empty. A node $x$ whose copy is in the searching paths constructed in the first $j$ iterations can never appear in $L$ in the 
$(j+1)$-th iteration because it has been removed from $D_{F}$. It follows that the searching paths constructed in the $(j+1)$-th iteration are node-disjoint with the searching paths constructed in the first $j$ iterations. Thus they are edge-disjoint. Since $L$ is empty, it is not difficult to see that the proof for the first iteration also holds for the $(j+1)$-th iteration. It follows that the searching paths constructed in the $(j+1)$-th iteration form a tree that is node disjoint with other $j$ trees belonging to the first $j$ iterations respectively, that is, these trees form a forest all together. By Lemma 7, $G$ consists of all searching paths constructed during the execution of NDTRV. It follows that $G$ is a forest. This completes the inductive proof for the whole procedure.

Lemma 9. $|V(G)|$ is equal to $\left|V\left(D_{F}\right)\right|$.

Proof. According to NDTRV, a node $v$ is added to $L$ if and only if its copy is added to $G$, and $v$ is removed from $L$ if and only if $v$ is removed from $D_{F}$. By the proof in Lemma 8, no node in $D_{F}$ can be added to $L$ more than once. By the proof of Lemma 6 , all nodes of $D_{F}$ are removed by NDTRV. It follows that all nodes in $D_{F}$ have exactly one copy in $G$. Thus $|V(G)|=\left|V\left(D_{F}\right)\right|$

We give the proof of Theorem 4 below.

Proof. (Theorem 4) In the procedure of constructing $G$ in Fig. 1, we construct a list $Q$ which consists of actions of type (i) and (ii). We claim that $Q$ is exactly the sequence satisfying the requirement of this theorem. By Lemma 7, the number of actions of type (ii) in $Q$ is exactly $\left|V\left(D_{F}\right)\right|=|V(G)|$. NDTRV adds an action of type (i) to $Q$ if and only if it adds an an edge to $E(G)$. Moreover, $Q$ and $E(G)$ are both empty at the beginning of NDTRV. It follows that there is a one-to-one correspondence between actions of type (i) in $Q$ and edges in $G$. By Lemma 8, $G$ is a forest. As a result, $|E(G)| \leq|V(G)|$, and the length of $Q$ is bounded by $|E(G)|+|V(G)| \leq 2|V(G)|=2\left|V\left(D_{F}\right)\right|$.

\subsection{The deterministic algorithm}

Now we are ready to give the deterministic algorithm FLIPDT for the Parameterized Flip Distance problem. The specific algorithm is presented in Fig. 3. As mentioned above, we assume that NDTRV is always able to guess the optimal choice correctly. In fact, FLIPDT achieves this by trying all possible sequences of actions and partitions of $k$. At the top level, FLIPDT branches into all partitions of $k$, namely $\left(k_{1}, \ldots, k_{t}\right)$ satisfying $k_{1}+\ldots+k_{t}=k$ and $k_{1}, \ldots, k_{t} \geq 1$, in which $k_{i}(i=1, \ldots, t)$ equals the size of the track tree $A_{i}$ constructed during the $i$-th iteration.

Suppose that FLIPDT is under some partition $\left(k_{1}, \ldots, k_{t}\right)$. Let $T_{\text {iteration }}^{0}=T_{\text {start }}$. FLIPDT permutates all necessary edges in $T_{\text {start }}$ in the lexicographical order, and the ordering is denoted by $O_{\text {lex }}$. Here we number the given points of $\mathcal{P}$ in the Euclidean plane from 1 to $n$ arbitrarily and label one edge by a tuple consisting of two numbers of its endpoints (the smaller number is ahead of the other one). Thus we can order the edges lexicographically. FLIPDT performs $t$ iterations. At the beginning of the $i$-th iteration, $i=1, \ldots, t$, we denote the current triangulation by $T_{\text {iteration }}^{i-1}$. For $i=1, \ldots, t, T_{\text {iteration }}^{i}$ is also the triangulation resulting from the execution of the $i$-th iteration. At the beginning of the $i$-th iteration $(i=1, \ldots, t)$, FLIPDT repeatedly picks the next edge in $O_{\text {lex }}$ until finding a necessary edge $e$ belonging to $T_{\text {iteration }}^{i-1}$ (just pick the first edge in $O_{l e x}$ in the first iteration). Note that one edge in $O_{l e x}$ may not be a necessary edge anymore with respect to $T_{\text {iteration }}^{i-1}$. Moreover, if FLIPDT reaches the end of $O_{l e x}$ but does not find a necessary edge belonging 
$\operatorname{FLIPDT}\left(T_{\text {start }}, T_{\text {end }}, k\right)$

Input: two triangulations $T_{\text {start }}$ and $T_{\text {end }}$ of a point set $\mathcal{P}$ in the Euclidean plane and an integer $k$.

Output: return YES if there exists a sequence of flips of length $k$ that transforms $T_{\text {start }}$ into $T_{\text {end }}$; otherwise return NO.

1. For each partition $\left(k_{1}, \ldots, k_{t}\right)$ of $k$ satisfying $k_{1}+k_{2}+\ldots+k_{t}=k$ and $k_{1}, \ldots, k_{t} \geq 1$ do

1.1 Order all necessary edges in $T_{\text {start }}$ lexicographically and denote this ordering by $O_{\text {lex }}$;

$1.2 \quad \operatorname{FDSearch}\left(T_{\text {start }}, 1,\left(k_{1}, \ldots, k_{t}\right)\right) ; /{ }^{*}$ iteration 1 distributed with $k_{1} * /$

2. Return NO;

$\operatorname{FDSearch}\left(T, i,\left(k_{1}, \ldots, k_{t}\right)\right) \quad{ }^{*}$ the concrete branching procedure*/

Input: a triangulation $T$, an integer $i$ denoting that the algorithm is at the $i$-th iteration and a partition $\left(k_{1}, \ldots, k_{t}\right)$ of $k$.

Output: return YES if the instance is accepted.

1. Repeatedly pick the next edge in $O_{l e x}$ until finding a necessary edge $e$ with respect to $T$ and $T_{\text {end }}$;

2. If it reaches the end of $O_{l e x}$ but finds no necessary edge in $T$ then

2.1 Update $O_{\text {lex }}$ by permutating all necessary edges in $T_{\text {iteration }}^{i-1}$ in lexicographical order, and pick the first edge $e$ in $O_{l e x}$;

3. For each possible sequence of actions $s e q_{i}$ of length $2 k_{i}-1$ do

$3.1 \quad T^{\prime}=\operatorname{Transform}\left(T, \operatorname{seq}_{i}, e\right)$;

3.2 If $i<t$ then $\quad /{ }^{*}$ continue to the next iteration distributed with $k_{i+1} * /$

3.2.1 $\operatorname{FDSearch}\left(T^{\prime}, i+1,\left(k_{1}, \ldots, k_{t}\right)\right)$;

$3.3 \quad$ Else if $i=t$ and $T^{\prime}=T_{\text {end }}$ then $\quad /{ }^{*}$ compare $T^{\prime}$ with $T_{\text {end }} * /$

3.3.1 Return YES;

$\operatorname{Transform}(T, s, e) \quad /{ }^{*}$ subprocess for transforming triangulations*/

Input: a triangulation $T$, a sequence of actions $s$ and a starting edges $e$.

Output: a new triangulation $T^{\prime}$.

1. Perform a sequence of actions $s$ starting from $e$ in $T$, getting a new triangulation $T^{\prime}$;

2. Return $T^{\prime}$;

Figure 3 The deterministic algorithm for the Flip Distance problem

to $T_{\text {iteration }}^{i-1}$, it needs to update $O_{l e x}$ by clearing $O_{l e x}$ and permutating all necessary edges in $T_{\text {iteration }}^{i-1}$ lexicographically, and choose the first edge in the updated ordering $O_{\text {lex }}$. Then FLIPDT branches into every possible sequence of actions $s e q_{i}$ of length $2 k_{i}-1$. For each enumeration of $s e q_{i}$, FLIPDT performs the actions of $s e q_{i}$ on $T_{i \text { teration }}^{i-1}$ starting from $e$ and gets a new triangulation $T_{\text {iteration. }}^{i}$ For every triangulation $T_{\text {iteration }}^{i}$ resulting from $s e q_{i}$, FLIPDT performs the $(i+1)$-th iteration on $T_{\text {iteration }}^{i}$ FLIPDT proceeds as above from the first iteration to the last iteration. When FLIPDT finishes the last iteration, it judges if the resulting triangulation $T_{\text {iteration }}^{t}$ is equal to $T_{\text {end }}$. If they are equal, the input instance is a yes-instance. Otherwise, FLIPDT rejects this case and proceeds.

Now we analyse how to enumerate all possible sequences of length $2 k_{i}-1$. By the proof of Lemma 8 and Theorem 4, the searching paths constructed during each iteration form a track tree in which a node corresponds to an action of type (ii) while an edge corresponds to an action of type (i). It follows that the number of actions of type (ii) is $k_{i}$, and the number of actions of type (i) is $k_{i}-1$ since the number of nodes equals the number of edges plus one in a tree. According to NDTRV, the last action $\gamma$ in $s e q_{i}$ must be of type (ii), and in any prefix of $s e q_{i}-\gamma$ the number of actions of type (i) must not be less than that of type (ii). Thus FLIPDT only needs to enumerate all sequences of length $2 k_{i}-1$ satisfying the above constraints.

The following theorem proves the correctness of the algorithm FLIPDT. 
- Theorem 10. Let $\left(T_{\text {start }}, T_{\text {end }}, k\right)$ be an input instance. FLIPDT is correct and runs in time $O^{*}\left(k \cdot 32^{k}\right)$.

Proof. Suppose that $\left(T_{\text {start }}, T_{\text {end }}, k\right)$ is a yes-instance. There must exist a sequence of flips $F$ of length $k$ such that $T_{\text {start }} \stackrel{F}{\rightarrow} T_{\text {end }}$. Thus $D_{F}$ exists according to Definition 1 . By NDTRV, Lemma 7 and Lemma 8, there exists an undirected graph $G$ consisting of a set of node-disjoint track trees $A_{1}, \ldots, A_{t}$. Moreover, Theorem 4 shows that there exists a sequence of actions $Q$ following which we can perform all flips of $D_{F}$ in a topologically-sorted order. Due to NDTRV, $Q$ consists of several subsequences $s e q_{1}, \ldots, s e q_{t}$, in which $s e q_{i}$ is constructed in the $i$-th iteration and corresponds to the track tree $A_{i}$ for $i=1, \ldots, t$. Supposing the size of $A_{i}$ is $\lambda_{i}$ for $i=1, \ldots, t$ satisfying $\lambda_{1}+\ldots+\lambda_{t}=k$, seq $_{i}$ contains $\lambda_{i}$ actions of type (ii) corresponding to the nodes of $A_{i}$ as well as $\lambda_{i}-1$ actions of type (i) corresponding to the edges of $A_{i}$. FLIPDT guesses the size of every track tree by enumerating all possible partitions of $k$ into $\left(k_{1}, \ldots, k_{t}\right)$ such that $k_{1}+\ldots+k_{t}=k$ and $k_{1}, \ldots, k_{t} \geq 1$. We say that $k_{i}$ is distributed to the $i$-th iteration or the distribution for the $i$-th iteration is $k_{i}$ for $i=1, \ldots, t$.

We claim that FLIPDT is able to perform a sequence $\Sigma$ of actions which correctly guesses every subsequence $s e q_{1}, \ldots, s e q_{t}$ of the objective sequence $Q$, that is, $\Sigma$ is a concatenation of $s e q_{1}, \ldots, s e q_{t}$. Suppose that FLIPDT has completed $i$ iterations. We prove this claim by induction on $i$. At the first iteration, FLIPDT starts by picking the first necessary edge $e_{1}$ in list $O_{l e x}$. In the first iteration of constructing $Q$, NDTRV starts by picking an arbitrary necessary edge. Without loss of generality, it chooses $e_{1}$ and construct $s e q_{1}$ starting from $e_{1}$. The length of $s e q_{1}$ is $2 \lambda_{1}-1$. Since FLIPDT tries every distribution in $\{1, \ldots, k\}$ for the first iteration and $1 \leq \lambda_{1} \leq k$, there is a correct guess of the distribution equal to $\lambda_{1}$ for this iteration. Under this correct guess, FLIPDT tries all possible sequences of actions of length $2 \lambda_{1}-1$ starting from $e_{1}$. It follows that FLIPDT is able to perform a sequence that is equals to $s e q_{1}$ in the first iteration resulting in a triangulation $T_{1}$.

Suppose that the claim is true for any first $i$ iterations $(1 \leq i<t)$. That is, under some guess for the partition of $k, \lambda_{1}, \ldots, \lambda_{i}$ are distributed to the first $i$ iterations respectively. Moreover, FLIPDT has completed $i$ iterations and performed a sequence of actions seq $_{\text {concat }, i}$, which is equal to the concatenation of $s e q_{1}, \ldots, s e q_{i}$, resulting in a triangulation $T_{i}$. Based on $T_{i}$ and $s e q_{\text {concat }, i}$, FLIPDT is ready to perform the $(i+1)$-th iteration. Suppose that FLIPDT picks $e_{i+1}$ from $O_{l e x}$. Let us see the construction of $Q$ in NDTRV. Suppose NDTRV has constructed the first $i$ track trees $A_{1}, \ldots, A_{i}$, and it is ready to begin a new iteration by arbitrarily picking a necessary edge in the current triangulation. Since FLIPDT correctly guessed and performed the first $i$ subsequences of $Q, T_{i}$ is exactly equal to the current triangulation in NDTRV. Thus $e_{i+1}$ is a candidate edge belonging to the set of all selectable necessary edges for NDTRV in this iteration. Without loss of generality, it chooses $e_{i+1}$ and constructs $s e q_{i+1}$ of length $2 \lambda_{i+1}-1$ starting from $e_{i+1}$. Since the sizes of $A_{1}, \ldots, A_{i}$ are $\lambda_{1}, \ldots, \lambda_{i}$ respectively, we get that $1 \leq \lambda_{i+1} \leq k-\left(\lambda_{1}+\ldots+\lambda_{i}\right)$. We argue that FLIPDT is able to perform a sequence that is equal to the concatenation of $s e q_{1}, \ldots, s e q_{i+1}$. Since the edges in $O_{\text {lex }}$ are ordered lexicographically and FLIPDT chooses necessary edges in a fixed manner, FLIPDT is sure to choose $e_{i+1}$ to begin the $(i+1)$-th iteration for every guessed sequence in which the first $i$ subsequences are equal to $s e q_{1}, \ldots, s e q_{i}$ respectively. Thus FLIPDT actually tries every distribution in $\left\{1, \ldots, k-\left(\lambda_{1}+\ldots+\lambda_{i}\right\}\right)$ for the $(i+1)$-th iteration starting from $e_{i+1}$ based on $T_{i}$ and $s e q_{\text {concat }, i}$. It follows that there is a correct guess of distribution for the $(i+1)$-th iteration which is equal to $\lambda_{i+1}$. Under this correct guess of distribution, FLIPDT tries all possible sequences of length $2 \lambda_{i+1}-1$ starting from $e_{i+1}$ on $T_{i}$ based on $s e q_{\text {concat }, i}$, ensuring that one of them is equal to $s e q_{i+1}$. It follows that the claim is true for the first $i+1$ iterations. This completes the inductive proof for the claim. 
If ( $\left.T_{\text {start }}, T_{\text {end }}, k\right)$ is a yes-instance, the action sequence $Q$ of length at most $2 k$ exists and the deterministic algorithm can find such a sequence. Otherwise, there is no valid sequence $F$ of length $k$. Thus there is no such action sequence $Q$. As a result, FLIPDT returns NO. It is proved that FLIPDT decides the given instance $\left(T_{\text {start }}, T_{\text {end }}, k\right)$ correctly.

Finding and ordering all necessary edges in $T_{\text {start }}$ takes $O(n+k \log k)$ time, and FLIPDT may update the ordering $O_{l e x}$ at the beginning of each iteration. The number of partitions of $k$ is known as the composition number of $k$, which is $2^{k-1}$. Under each partition $\left(k_{1}, \ldots, k_{t}\right)$ of $k$ and for each $k_{i}, i=1, \ldots, t$, we enumerate all possible subsequences of actions in which there are $k_{i}$ actions of type (ii) and $k_{i}-1$ actions of type (i). It follows that the number of all possible subsequences is bounded by $\left(\begin{array}{c}2\left(k_{i}-1\right) \\ k_{i}-1\end{array}\right) \times 4^{k_{i}-1}=O^{*}\left(16^{k_{i}}\right)$ since there are four choices for action (i) and one choice for action (ii). Here we use Stirling's approximation $n ! \approx \sqrt{2 \pi n}(n / e)^{n}$ and get that $\left(\begin{array}{c}2\left(k_{i}-1\right) \\ k_{i}-1\end{array}\right)=O^{*}\left(4^{k_{i}}\right)$. It follows that there are $O^{*}\left(16^{k_{1}}\right) \times O^{*}\left(16^{k_{2}}\right) \times \ldots \times O^{*}\left(16^{k_{t}}\right)=O^{*}\left(16^{k}\right)$ cases under each partition. Since for each case we can perform the sequence of actions in $O(k)$ time, and the resulting triangulation can be compared to $T_{\text {end }}$ in $O(k)$ time, the running time of the whole algorithm is bounded by $O^{*}\left(k \cdot 2^{k-1} \cdot(n+k \log k)+k \cdot 2^{k-1} \cdot 16^{k}\right)=O^{*}\left(k \cdot 32^{k}\right)$.

According to the definition of the Flip Distance problem, we need to decide if we can find a shorter valid flip sequence for the given triangulations $T_{\text {start }}$ and $T_{\text {end }}$. This is achieved by calling FLIPDT on each instance $\left(T_{\text {start }}, T_{\text {end }}, k^{\prime}\right)$ for $k^{\prime}=0, \ldots, k$. The running time is bounded by $\sum_{k^{\prime}=0}^{k} O^{*}\left(k^{\prime} \cdot 32^{k^{\prime}}\right)=O^{*}\left(k \cdot 32^{k}\right)$.

\section{Conclusion}

In this paper we presented an FPT algorithm running in time $O^{*}\left(k \cdot 32^{k}\right)$ for the Parameterized Flip Distance problem, improving the previous $O^{*}\left(k \cdot c^{k}\right)$-time $\left(c \leq 2 \times 14^{11}\right)$ FPT algorithm by Kanj and Xia [15]. An important related problem is computing the flip distance between triangulations of a convex polygon, whose traditional complexity is still unknown. Although our algorithm can be applied to the case of convex polygon, it seems that an $O\left(c^{k}\right)$ algorithm with smaller $c$ for this case probably exists due to its more restrictive geometric property. In addition, whether there exists a polynomial kernel for the Parameterized Flip Distance problem is also an attractive problem.

\section{References}

1 Oswin Aichholzer, Ferran Hurtado, and Marc Noy. A lower bound on the number of triangulations of planar point sets. Computational Geometry, 29(2):135-145, 2004. doi: $10.1016 / \mathrm{j}$. comgeo.2004.02.003.

2 Oswin Aichholzer, Wolfgang Mulzer, and Alexander Pilz. Flip distance between triangulations of a simple polygon is NP-complete. Discrete \& Computational Geometry, 54(2):368389, 2015. doi:10.1007/s00454-015-9709-7.

3 Hans L. Bodlaender, Rodney G. Downey, Michael R. Fellows, Michael T. Hallett, and Harold T. Wareham. Parameterized complexity analysis in computational biology. Computer Applications in the Biosciences, 11(1):49-57, 1995. doi:10.1093/bioinformatics/11.1. 49.

4 Jianer Chen. Parameterized computation and complexity: A new approach dealing with nphardness. J. Comput. Sci. Technol., 20(1):18-37, 2005. doi:10.1007/s11390-005-0003-7.

5 Jianer Chen, Donald K. Friesen, Weijia Jia, and Iyad A. Kanj. Using nondeterminism to design efficient deterministic algorithms. Algorithmica, 40(2):83-97, 2004. doi:10.1007/ s00453-004-1096-z. 
6 Jianer Chen, Chao Xu, and Jianxin Wang. Dealing with 4-variables by resolution: An improved maxsat algorithm. Theor. Comput. Sci., 670:33-44, 2017. doi:10.1016/j.tcs. 2017.01 .020 .

7 Marek Cygan, Fedor V. Fomin, Lukasz Kowalik, Daniel Lokshtanov, Dániel Marx, Marcin Pilipczuk, Michal Pilipczuk, and Saket Saurabh. Parameterized Algorithms. Springer, 2015. doi : 10.1007/978-3-319-21275-3.

8 Marek Cygan, Jesper Nederlof, Marcin Pilipczuk, Michal Pilipczuk, Johan M. M. van Rooij, and Jakub Onufry Wojtaszczyk. Solving connectivity problems parameterized by treewidth in single exponential time. In Proceedings of the 52nd Annual IEEE Symposium on Foundations of Computer Science, FOCS, Palm Springs, USA, pages 150-159, 2011. doi:10.1109/FOCS.2011.23.

9 Marek Cygan, Marcin Pilipczuk, Michal Pilipczuk, and Jakub Onufry Wojtaszczyk. Subset feedback vertex set is fixed-parameter tractable. SIAM J. Discrete Math., 27(1):290-309, 2013. doi:10.1137/110843071.

10 Mark de Berg, Otfried Cheong, Marc J. van Kreveld, and Mark H. Overmars. Computational geometry: algorithms and applications, 3rd Edition. Springer, 2008.

11 Gerald E. Farin. Curves and surfaces for computer-aided geometric design - a practical guide (4. ed.). Computer science and scientific computing. Academic Press, 1997.

12 Fedor V. Fomin, Daniel Lokshtanov, and Saket Saurabh. Efficient computation of representative sets with applications in parameterized and exact algorithms. In Proceedings of the Twenty-Fifth Annual ACM-SIAM Symposium on Discrete Algorithms, SODA, Portland, USA, pages 142-151, 2014. doi:10.1137/1.9781611973402.10.

13 Bernd Hamann. Modeling contours of trivariatc data. Mathematical Modeling and Numerical Analysis, 26:51-75, 1992.

14 Ferran Hurtado, Marc Noy, and Jorge Urrutia. Flipping edges in triangulations. Discrete \& Computational Geometry, 22(3):333-346, 1999. doi:10.1007/PL00009464.

15 Iyad A. Kanj and Ge Xia. Flip Distance is in FPT time $O\left(n+k \cdot c^{k}\right)$. In proceedings of the 32nd International Symposium on Theoretical Aspects of Computer Science, STACS, Garching, Germany, pages 500-512, 2015. doi:10.4230/LIPIcs.STACS.2015.500.

16 Iyad A. Kanj and Ge Xia. Computing the flip distance between triangulations. to appear in Discrete \& Computational Geometry, 2017.

17 Charles L. Lawson. Transforming triangulations. Discrete Mathematics, 3(4):365-372, 1972. doi:10.1016/0012-365X (72)90093-3.

18 Anna Lubiw and Vinayak Pathak. Flip distance between two triangulations of a point set is NP-complete. Computational Geometry, 49:17-23, 2015. doi:10.1016/j.comgeo. 2014. 11.001 .

19 Joan M. Lucas. An improved kernel size for rotation distance in binary trees. Information Processing Letters, 110(12-13):481-484, 2010. doi:10.1016/j.ipl.2010.04.022.

20 Alexander Pilz. Flip distance between triangulations of a planar point set is APX-hard. Computational Geometry, 47(5):589-604, 2014. doi:10.1016/j.comgeo.2014.01.001.

21 Larry L. Schumaker. Triangulations in CAGD. IEEE Computer Graphics and Applications, 13(1):47-52, 1993. doi:10.1109/38.180117.

22 Feng Shi, Jianxin Wang, Yufei Yang, Qilong Feng, Weilong Li, and Jianer Chen. A fixed-parameter algorithm for the maximum agreement forest problem on multifurcating trees. SCIENCE CHINA Information Sciences, 59(1):1-14, 2016. doi:10.1007/ s11432-015-5355-1.

23 Daniel D. Sleator, Robert E. Tarjan, and William P. Thurston. Rotation distance, triangulations, and hyperbolic geometry. In Proceedings of the 18th Annual ACM Symposium on Theory of Computing, STOC, Berkeley, USA, pages 122-135, 1986 . doi: 10.1145/12130.12143. 